\title{
Predictors of Mortality in Patients with
}

\section{Carbapenem-Resistant Gram-Negative Bacilli or Vancomycin-Resistant Enterococci Bacteremia}

This article was published in the following Dove Press journal: Infection and Drug Resistance

Hye Jin Shi

Jin Seo Lee $\mathbb{1}^{2}$

Yong Kyun Cho'

Joong Sik Eom (D)

'Division of Infectious Diseases, Department of Internal Medicine, Gil Medical Center, Gachon University School of Medicine, Incheon, Republic of Korea; ${ }^{2}$ Division of Infectious Diseases, Department of Internal Medicine, Kangdong Sacred Hospital, Hallym University School of Medicine, Seoul, Republic of Korea
Correspondence: Joong Sik Eom Division of Infectious Diseases, Department of Internal Medicine, Gil Medical Center, Gachon University School of Medicine, 774 Namdongdaero, Namdong-Gu, Incheon 21565, South Korea

Tel $+82-32-460-2630$

Fax +82-32-460-4884

Email helppl@gachon.ac.kr
Purpose: The incidences of carbapenem-resistant gram-negative bacilli (CRGNB) and vancomycin-resistant Enterococci (VRE) have increased rapidly in South Korea since 2000. The mortality rate for CRGNB or VRE bacteremia cases is higher than that for nonresistant bacteremia cases. The factors associated with higher mortality are unclear. We investigated the factors associated with mortality from CRGNB or VRE bacteremia and compared the relative risk of these factors.

Patients and Methods: We retrospectively collected data from adult patients with CRGNB or VRE bacteremia. Patients were grouped according to whether they survived or died. The data from both groups were compared.

Results: During the study period, 171 cases of CRGNB or VRE bacteremia were identified, of which 100 were CRGNB bacteremia cases and 71 were VRE bacteremia cases. Multivariate analysis revealed significant associations with Pitt bacteremia score (PBS) (odds ratio [OR] 1.329, 95\% confidence interval [CI] 1.049-1.684). In the multivariate analysis, negative conversion of follow-up blood culture (FUBC) was related with oneweek mortality from CRGNB or VRE bacteremia (OR 17.623, 95\% CI 5.726-54.244). In the multivariate analysis of risk factors for 28-day mortality for CRGNB or VRE bacteremia, the significant risk factors were bacteremia of respiratory origin (OR 4.491, 95\% CI 1.62212.435) and positive FUBC (OR 4.082, 95\% CI 1.626-10.204).

Conclusion: Despite the high mortality rate in patients with CRGNB or VRE bacteremia, the related mortality could be predicted by independent risk factors of PBS, positive FUBC, and bacteremia of respiratory origin.

Keywords: prognostic factor, carbapenem-resistant, vancomycin-resistant, bacteremia, mortality

\section{Introduction}

Although introduced very recently on a global scale, active surveillance has revealed the rapidly increasing incidence of carbapenem-resistant gram-negative bacilli (CRGNB) ${ }^{1}$ and vancomycin-resistant Enterococci (VRE). VRE accounts for approximately $4 \%$ of all hospital-associated infections in the United States. ${ }^{2}$ The incidence of CRGNB in 2018 was approximately 0.30-2.93 infections per 100,000 people per year in the USA. ${ }^{3}$ In the Republic of Korea, the national surveillance system does not specifically monitor CRGNB. Nonetheless, an increased incidence of carbapenem-resistant gram Enterobacteriaceae (CRE) carriers from 5717 in 2017 to 14205 in 2019 were reported. $^{4}$ 
CRGNB and VRE strains are typically extensively drug-resistant. There are few available treatment options and those that are available have uncertain effectiveness and are highly toxic. ${ }^{5,6}$ Mortality among patients with CRGNB or VRE bacteremia is higher than that of patients with non-resistant bacteremia. ${ }^{7,8}$ However, the reason behind this higher mortality is unclear. Furthermore, in cases of CRGNB or VRE bacteremia, the factors associated with patient survival and death are unclear, despite their increasing importance.

Identification of these risk factors would be valuable in predicting or improving treatment outcomes for patients during the initial examination. In this study, we attempted to identify the factors associated with mortality due to CRGNB or VRE bacteremia and compared their relative risks.

\section{Patients and Methods}

\section{Study Design and Patient Population}

We retrospectively collected data from cases of CRGNB or VRE bacteremia at Gil Medical Center, a universityaffiliated, 1650-bed, tertiary-care hospital in Incheon, Republic of Korea, between January 2015 and June 2019. Patients older than 18 years were included in the study. We searched the electronic medical records using the ICD-10 $\operatorname{code}^{9}$ for eligible patients diagnosed with CRGNB or VRE bacteremia from the initial blood culture. Exclusion criteria included patients without bacteremia, obvious evidence of contamination (eg, consultation paper or no fever and no antibiotics), and no immediate follow-up (eg, transfer-out or follow-up loss after emergency room visit). This study was performed and described in accordance with the Strengthening the Reporting of Observational Studies in Epidemiology guidelines for cohort studies.

\section{Data Collection and Analyses}

Electronic medical records with the clinical and microbiological data of patients were reviewed and collected. The data included demographic information, source of infection, survival or death at one-week and 28 days, microorganisms isolated from blood cultures, antibiotic susceptibility, comorbidities (eg, hypertension, diabetes mellitus, acquired immunodeficiency syndrome, chronic kidney disease, and rheumatic disease), need for intensive care, timing of effective antibiotic uses, duration of antimicrobial therapy, invasive procedures, severity indices, and follow-up blood culture (FUBC) information. The patients were grouped according to whether they survived or died at one week and 28 days. We also collected the first laboratory data at the time of infection diagnosis. The data included white blood cell (WBC) count, segmented neutrophil percentage, absolute neutrophil count, hemoglobin, platelet count, erythrocyte sedimentation rate, international normalized ratio, protein, albumin, total bilirubin, aspartate aminotransferase, alanine aminotransferase, C-reactive protein (CRP), procalcitonin, lactic acid, blood urea nitrogen (BUN), and creatinine. Microbiological data were obtained from the database at our clinical microbiology laboratory.

Follow-up blood cultures (FUBCs) were defined by more than one separate blood culture taken more than 24 $\mathrm{h}$ after the initial blood culture. The duration of bacteremia (in days) among patients with FUBCs was calculated from the initial date of a positive culture to the latest date of a positive culture for the same bacteria, as long as the last set of positive cultures was examined at least $24 \mathrm{~h}$ after the initial culture. Initial blood culture was defined as the first positive blood culture at the time of infection. The number of FUBCs for each episode was recorded until negative conversion. Severity indices including Systemic Inflammatory Response Syndrome, Acute Physiologic Assessment and Chronic Health Evaluation (APACHE), Sequential Organ Failure Assessment (SOFA), Pitt bacteremia score (PBS), and Charlson comorbidity score of each patient were collected at the time of infection diagnosis. Antimicrobial susceptibility was identified using the MicroScan WalkAway 96 device (Siemens Healthcare Diagnostics, Deerfield, IL, USA) and the VITEK 2 device (bioMérieux, Marcy-l'Étoile, France), using Clinical and Laboratory Standards Institute criteria and guidelines. ${ }^{10}$

\section{Statistical Analyses}

Results are expressed as mean \pm standard deviation and as incidences in the study population. Normality test was performed for all continuous variables. Student's $t$-test and Mann-Whitney test were used to compare continuous variables. The chi-square test and Fisher's exact test were used for categorical variables. To identify risk factors for one-week or 28-day mortality, a logistic regression model was used as control for confounding variables. All Pvalues were two-tailed, and $\mathrm{P}<0.05$ were considered significant. Statistically significant variables in the univariate analyses were used as candidates for multivariate analysis in addition to the main variables of clinical 
importance. Collinearity test was also performed to exclude the possibility of cross-influences. To evaluate the appropriate cut-off values for laboratory data, such as CRP levels, a receiver-operating characteristic (ROC) analysis was performed. Risk factors are reported as odds ratios (ORs) and 95\% confidence intervals (CIs) for mortality, and mean difference and standard deviation (SD) are reported. When the median and interquartile ranges (IQRs) were reported, the median was assumed to reflect the mean and IQR was assumed to be 1.35 SD. IBM SPSS statistics software for Windows (version 24; SPSS Inc., Chicago, IL, USA) was used for the analyses.

\section{Results}

During the study period, 171 patients with CRGNB or VRE bacteremia were identified. One hundred were CRGNB bacteremia cases and 71 were VRE bacteremia cases. Baseline characteristics of patients are presented in Table 1 . The median age of the study population was 70 years $(I Q R=18)$ and $59.7 \%$ were male. The most common underlying diseases were hypertension, heart failure, and diabetes mellitus. In the microbiological analyses of causative pathogens, Acinetobacter baumannii (38\%) was the most common pathogen among CRGNB pathogens, followed by Klebsiella pneumoniae (15.2\%), Pseudomonas aeruginosa (7.6\%), and Escherichia coli (5.8\%).

Various severity indices were used to predict one-week and 28-day mortality. Univariate and multivariate analyses of the severity indexes are presented in Tables 2 and 3. All the variables except Charlson comorbidity index were related with one-week mortality in univariate analysis. Only PBS was statistically significant in the multivariate analysis (Table 2). All of the severity indices were related to 28-day mortality in the univariate analysis. None were significant in the multivariate analysis (Table 3 ).

Variables related to the one-week mortality due to CRGNB or VRE bacteremia were also analyzed. For clearer visualization and analysis, variables with consecutive numbers were converted to intervals using normal cutoff values. In addition, CRP was converted to intervals $(\geq 15 \mathrm{mg} / \mathrm{dL}$ ) using the area under the ROC curve. Hospitalization for longer than 90 days, respiratory origin bacteremia, central line-associated bloodstream infection, positive FUBC, initial hypotension, CRP $>15 \mathrm{mg} / \mathrm{dL}$, and severity indices (except for the Charlson comorbidity score) were significantly more common in patients who died than in survivors (Table 4). Other significantly different test results in the death and survivor groups included underlying liver cirrhosis $(21.5 \%$ vs $8.0 \%, \mathrm{P}=0.04)$, initial mechanical ventilation $(27.3 \%$ vs $48.0 \%, \mathrm{P}=0.01)$, platelet count $<150 \times 10^{3} \mathrm{~mm}^{3}(42.1 \%$ vs $74.0 \%, \mathrm{P}<0.01)$, and BUN $>22 \mathrm{mg} / \mathrm{dL}(49.6 \%$ vs $26 \%, \mathrm{P}<0.01)$.

Eight variables with lower P-values, including PBS and APACHE IV score, were analyzed via multivariate analysis. Only the negative conversion of FUBC was related with one-week mortality in patients with CRGNB or VRE bacteremia (Table 4).

Risk factors for 28-day mortality in CRGNB or VRE bacteremia were analyzed. Of the variables available at the initial patient evaluation, respiratory origin bacteremia, bacteremia of non-genitourinary origin, active treatment beginning after $48 \mathrm{~h}$, initial hypotension, CRP $\geq 15 \mathrm{mg}$ / $\mathrm{dL}$, albumin $<3.5 \mathrm{~g} / \mathrm{dL}$, and all severity indices were relative risk factors of 28-day mortality in the univariate analysis, whereas negative conversion of FUBC was a significant opposite factor of 28-day mortality (Table 5).

Initial neutropenia status $(8.2 \%$ vs $19.4 \%, \mathrm{P}=0.04)$, initial mechanical ventilation $(23.3 \%$ vs $37.7 \%, \mathrm{P}=0.01)$, initial $\mathrm{WBC}>120 \times 10^{3} \mathrm{~mm}^{3}$ or $<4 \times 10^{3} \mathrm{~mm}^{3}(50.7 \%$ vs $66.3 \%, \mathrm{P}=0.04)$, platelet count $<150 \times 10^{3} \mathrm{~mm}^{3}$ (41.1\% vs $59.2 \%, \mathrm{P}=0.02)$, creatinine $>1.2 \mathrm{mg} / \mathrm{dL}$, and $\mathrm{BUN}>22 \mathrm{mg} /$ $\mathrm{dL}(43.8 \%$ vs $67.3 \%, \mathrm{P}<0.01)$ were also significant factors associated with 28-day mortality in the univariate analysis (data not shown).

Considering the study population, nine variables with lower P-values, including PBS and APACHE IV score, were analyzed using multivariate analysis. In the multivariate analysis for 28-day mortality, bacteremia of respiratory origin and negative conversion of FUBC were significant positive and negative risk factors for CRGNB or VRE bacteremia-related mortality, respectively.

\section{Discussion}

Patients with VRE or CRGNB bacteremia have relatively higher mortality rates than patients with VSE or nonCRGNB bacteremia. ${ }^{11-13}$ Studies of VRE bacteremia have documented variable mortality rates. ${ }^{7,14,15}$ Moreover, 28-day mortality from CRGNB infection in China was $33.5 \%^{11,16,17}$ and a multicenter study in the USA reported a 30-day mortality rate of $49 \%$ and 14 -day mortality rate of $22 \% .{ }^{18}$ Our study population had 28 -day mortality rates of $42.7 \%$ and $29.2 \%$ for VRE and CRGNB, respectively, which are similar to previous studies. ${ }^{7,17}$

While all of the mentioned severity scores seemed to be related to 28-day mortality in the univariate analysis, no specific severity index was associated with 28-day 
Table I Baseline Characteristics of the Study Population $(N=\mid 7 I)$

\begin{tabular}{|c|c|c|c|}
\hline & \multirow[t]{2}{*}{ Number (\%) Median \pm IQR } & \multirow[b]{2}{*}{ Gastrointestinal Origin } & \multirow{2}{*}{$\begin{array}{l}\text { Number (\%) Median } \pm \text { IQR } \\
67(39.2 \%)\end{array}$} \\
\hline & & & \\
\hline Age & $70 \pm 18$ & Respiratory origin & $50(29.2 \%)$ \\
\hline Male sex & $102(59.7 \%)$ & CLABSI & 81 (47.4\%) \\
\hline CRGNB & $100(58.4 \%)$ & Genitourinary origin & 19 (II.1\%) \\
\hline \multirow[t]{2}{*}{ Hospitalized within 3 months } & $89(52.0 \%)$ & SSTI & 14 (8.2\%) \\
\hline & & CNS origin & $7(4.1 \%)$ \\
\hline HD & $40 \pm 46$ & APS & $59 \pm 35$ \\
\hline Length of ICU stay (days) & $24.52 \pm 62.43$ & APACHE IV score & $78 \pm 35$ \\
\hline FUBC negative conversion & 114 (66.7\%) & SOFA score & $9 \pm 4.5$ \\
\hline Underlying disorder & & PBS & $2.96 \pm 3.55$ \\
\hline Bed-ridden patient & 47 (27.5\%) & Charlson comorbidity index & $6.67 \pm 3.06$ \\
\hline Diabetes mellitus & $66(38.6 \%)$ & Laboratory data (units) & \\
\hline Hypertension & 87 (50.9\%) & WBC $\left(\times 10^{3} / \mu \mathrm{L}\right)$ & $12.22 \pm 9.81$ \\
\hline Heart failure & 87 (50.9\%) & Neutrophil count $\left(\times 10^{3} / \mu \mathrm{L}\right)$ & 117.48 \\
\hline Coronary disease & 19 (II.1\%) & Platelet $\left(\times 10^{3} / \mu \mathrm{L}\right)$ & $163.5 \pm 134.0$ \\
\hline Solid tumor & $62(3.3 \%)$ & Prothrombin time (INR) & $1.68 \pm 1.41$ \\
\hline Hematologic tumor & $26(15.2 \%)$ & Albumin (g/dL) & $2.63 \pm 0.57$ \\
\hline Neutropenia & $25(14.6 \%)$ & BUN (mg/dL) & $34.40 \pm 26.9$ \\
\hline End stage renal disorder & $16(9.4 \%)$ & Creatinine $(\mathrm{mg} / \mathrm{dL})$ & $1.50 \pm 1.45$ \\
\hline COPD & $24(14 \%)$ & Total bilirubin (mg/dL) & $3.88 \pm 6.27$ \\
\hline Liver cirrhosis & $30(17.5 \%)$ & Sodium & $|36.50 \pm 6.9|$ \\
\hline Solid organ transplantation & $5(2.9 \%)$ & Potassium & $3.86 \pm 0.66$ \\
\hline HSCT & $6(3.5 \%)$ & C-reactive protein (mg/dL) & $13.89 \pm 12.11$ \\
\hline Rheumatic disorder & 19 (II.1\%) & $\operatorname{ESR}(\mathrm{mm} / \mathrm{h})$ & $33.43 \pm 18.29$ \\
\hline Cerebrovascular accident & $49(28.7 \%)$ & Procalcitonin (mg/dL) & $13.98 \pm 24.87$ \\
\hline
\end{tabular}

Note: *Effective percent excluding missing values.

Abbreviations: N, number; IQR, interquartile range; CRGNB, carbapenem-resistant gram-negative bacilli; HD, hospital days; IQR, interquartile range; ICU, intensive care unit; FUBC, follow-up blood culture; COPD, chronic obstructive pulmonary disease; HSCT, hematopoietic stem cell transplantation; CLABSI, central line associated blood stream infection; SSTI, skin and soft tissue infection; CNS, central nerve system; APS, Admission Point Score; APACHE, Acute Physiology and Chronic Health Evaluation; SOFA, Sequential Organ Failure Assessment; PBS, Pitt bacteremia score; WBC, white blood cell; BUN, blood urea nitrogen; ESR, erythrocyte sedimentation rate.

Table 2 Severity Indexes and One-Week Mortality Data from Univariate and Multivariate Analyses

\begin{tabular}{|c|c|c|c|c|c|}
\hline \multirow[t]{2}{*}{ Severity Score } & \multicolumn{3}{|l|}{ Univariate Analysis } & \multicolumn{2}{|c|}{ Multivariate Analysis } \\
\hline & Alive (Mean \pm SD) & Death (Mean \pm SD) & P-value & $\operatorname{Exp}(B)$ & $95 \% \mathrm{Cl}$ \\
\hline APACHE IV score & $74.63 \pm 21.04$ & $106.18 \pm 29.85$ & 0.00 & $\mathrm{I} .04 \mathrm{I}$ & $0.995-1.089$ \\
\hline APACHE mortality rate & $44.30 \pm 26.47$ & $71.39 \pm 18.10$ & 0.00 & 0.974 & $0.928-1.023$ \\
\hline SOFA score & $7.38 \pm 4.16$ & $11.92 \pm 3.59$ & 0.00 & 1.151 & $0.97 \mid-1.365$ \\
\hline PBS & $1.88 \pm 2.67$ & $25.58 \pm 4.07$ & 0.00 & 1.316 & $1.026-1.688$ \\
\hline SIRS & $2.09 \pm 1.12$ & $2.76 \pm 1.10$ & 0.00 & 1.016 & $0.586-1.762$ \\
\hline Charlson comorbidity index & $66.52 \pm 3.06$ & $7.02 \pm 3.07$ & 0.33 & NA & NA \\
\hline
\end{tabular}

Abbreviations: SD, standard deviation; Exp (B), odds ratio; Cl, confidence interval; APACHE, Acute Physiology and Chronic Health Evaluation; SOFA, Sequential Organ Failure Assessment; PBS, Pitt bacteremia score; SIRS, systemic inflammatory response syndrome; NA, not available.

mortality in the multivariate analysis. One possible explanation for these observations is that the 28-day mortality from patients with CRGNB or VRE bacteremia was a multifactorial event, and therefore, initial severity indexes alone were insufficient to predict 28-day mortality. There are only a few previous studies concerning mortality in patients with CRE or VRE. In one study on VRE bacteremia, both a higher APACHE II score and the presence of septic shock at the onset of bacteremia were independent risk factors for all-cause mortality. ${ }^{13}$ Our study, which involved a larger number of patients and included patients with both CRGNB and VRE bacteremia, also indicated 
Table 3 Severity Indices and 28-Day Mortality Data from Univariate and Multivariate Analyses

\begin{tabular}{|c|c|c|c|c|c|}
\hline \multirow[t]{2}{*}{ Severity Score } & \multicolumn{3}{|l|}{ Univariate Analysis } & \multicolumn{2}{|c|}{ Multivariate Analysis } \\
\hline & Alive (Mean \pm SD) & Death (Mean \pm SD) & P-value & $\operatorname{Exp}(B)$ & $95 \% \mathrm{Cl}$ \\
\hline APACHE IV & $73.07 \pm 18.75$ & $91.89 \pm 30.78$ & 0.000 & 1.011 & $0.981-1.042$ \\
\hline SOFA score & $6.84 \pm 4.32$ & $10.37 \pm 04.13$ & 0.000 & 1.106 & $0.950-1.288$ \\
\hline PBS & $1.71 \pm 2.53$ & $3.91 \pm 3.92$ & 0.000 & 1.201 & $0.975-1.479$ \\
\hline SIRS & $2.04 \pm 1.23$ & $2.47 \pm 1.16$ & 0.021 & 0.794 & $0.496-1.269$ \\
\hline Charlson comorbidity index & $6.03 \pm 2.90$ & $7.14 \pm 3.11$ & 0.017 & 1.178 & $0.981-1.416$ \\
\hline
\end{tabular}

Abbreviations: IQR, interquartile range; Exp (B), odds ratio; Cl, confidence interval; APACHE, Acute Physiology and Chronic Health Evaluation; SOFA, Sequential Organ Failure Assessment; PBS, Pitt bacteremia score; SIRS, systemic inflammatory response syndrome.

Table 4 Factors Associated with One-Week Mortality from CRE ${ }^{\mathrm{a}}$ or VRE ${ }^{\mathrm{b}}$ Bacteremia (Univariate and Multivariate Analyses)

\begin{tabular}{|c|c|c|c|c|c|}
\hline \multirow[t]{2}{*}{ Severity Score } & \multicolumn{3}{|c|}{ Univariate Analysis } & \multicolumn{2}{|c|}{ Multivariate Analysis } \\
\hline & Alive & Death & P-value & $\operatorname{Exp}(B)$ & $95 \% \mathrm{Cl}$ \\
\hline Hospitalized over 90 days & $25(20.7 \%)$ & $4(8.0 \%)$ & 0.05 & 0.267 & $0.046-1.544$ \\
\hline Respiratory origin & $28(23.1 \%)$ & $22(44.0 \%)$ & 0.01 & 1.060 & $0.276-4.702$ \\
\hline CLABSI & $65(53.7 \%)$ & $16(32.0 \%)$ & 0.01 & 0.369 & $0.112-1.215$ \\
\hline FUBC-negative conversion & $104(86.0 \%)$ & $10(20.4 \%)$ & $<0.01$ & 17.623 & $5.726-54.244$ \\
\hline Hypotension & $30(25.0 \%)$ & $32(64.0 \%)$ & $<0.01$ & 4.153 & $0.84 I-20.499$ \\
\hline $\mathrm{CRP} \geq 15(\mathrm{mg} / \mathrm{dL})$ & 38 (32.5\%) & $28(58.3 \%)$ & 0.01 & 3.051 & $0.98|-9.27|$ \\
\hline PITTS bacteremia score & $1.88 \pm 2.67$ & $5.58 \pm 4.07$ & $<0.01$ & 1.061 & $0.802-1.404$ \\
\hline APACHE IV score & $74.63 \pm 21.04$ & $106.18 \pm 29.85$ & $<0.01$ & 1.028 & $0.997-1.060$ \\
\hline
\end{tabular}

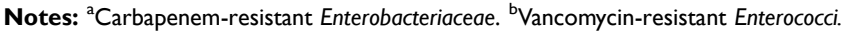

Abbreviations: $\operatorname{Exp}(B)$, odds ratio; $\mathrm{Cl}$, confidence interval; CLABSI, central line associated blood stream infection; FUBC, follow-up blood culture; CRP, C-reactive protein; APACHE, Acute Physiology and Chronic Health Evaluation.

Table 5 Factors Associated with 28-Day Mortality from CRE $^{\mathrm{a}}$ or VRE ${ }^{\mathrm{b}}$ Bacteremia (Univariate and Multivariate Analyses)

\begin{tabular}{|l|l|l|l|l|l|}
\hline \multirow{2}{*}{ Severity Score } & \multicolumn{2}{l|}{ Univariate Analysis } & \multicolumn{2}{l|}{ Multivariate Analysis } \\
\cline { 2 - 6 } & Alive Group & Death Group & P-value & Exp (B) & 95\% CI \\
\hline Respiratory origin & $10(13.7 \%)$ & $40(40.8 \%)$ & $<0.01$ & 4.491 & $1.622-12.435$ \\
Genitourinary origin & $13(17.8 \%)$ & $6(6.1 \%)$ & 0.02 & 0.659 & $0.204-2.127$ \\
FUBC negative-conversion & $64(87.7 \%)$ & $50(51.5 \%)$ & $<0.01$ & 0.245 & $0.098-0.615$ \\
Treatment began after 48 hours & $59(80.8 \%)$ & $81(82.7 \%)$ & 0.76 & NA & NA \\
Hypotension & $17(23.6 \%)$ & $45(45.9 \%)$ & $<0.01$ & 0.698 & $0.202-2.409$ \\
CRP $\geq 15 \mathrm{mg} / \mathrm{dL}$ & $21(30.0 \%)$ & $45(47.4 \%)$ & 0.02 & 2.063 & $0.940-4.527$ \\
Albumin <3.5 g/dL & $62(86.1 \%)$ & $95(96.9 \%)$ & 0.01 & 2.888 & $0.594-14.048$ \\
APACHE IV Score (mean \pm SD) & $73.07 \pm 18.75$ & $91.89 \pm 30.78$ & $<0.01$ & 1.015 & $0.993-1.037$ \\
PBS (mean \pm SD) & $1.71 \pm 2.53$ & $3.90 \pm 3.92$ & $<0.01$ & 1.053 & $0.848-1.309$ \\
\hline
\end{tabular}

Notes: a Carbapenem-resistant Enterobacteriaceae. ${ }^{b}$ Vancomycin-resistant Enterococci.

Abbreviations: Exp (B), odds ratio; Cl, confidence interval; CLABSI, central line associated blood stream infection; FUBC, follow-up blood culture; CRP, C-reactive protein; APACHE, Acute Physiology and Chronic Health Evaluation; PBS, Pitt bacteremia score; SD, standard deviation; NA, not available.

that initial PBS, FUBCs, and origin of bacteremia influence mortality. In another study of 90 patients, non-significant adverse outcomes appeared to be more likely among patients with previous carbapenem exposure and neutropenia and were related to the severity of septicemia and time to initiation of bloodstream infection. ${ }^{19}$ However, given the increased number of CRGNB or VRE carriers or bacteremia cases, we think that our study findings are credible and important.

A study on CRE bacteremia showed that KPC-3-Kp bacteremia and bacteremia onset in the intensive care unit were independently associated with mortality. ${ }^{11,19}$ Presently, we did not analyze the type of resistance. However, other studies reported no difference between 
carbapenemase-producing Enterobacteriaceae (CPE) and non-CPE infections. ${ }^{1,3}$ Moreover, we observed that positive FUBCs and PBS were significantly related with 28-day and one-week mortality from CRGNB or VRE bacteremia. There was a median of $47 \mathrm{~h}$ from bacteremia onset until active antimicrobial therapy in a previous study. ${ }^{18}$ Similarly, our study also demonstrated that only half of the patients $(52 \%)$ received effective antibiotics before 48 h. Despite a decade of multi-drug resistant (MDR) organism-related therapeutic development, there have been protracted delays in the treatment of CRGNB or VRE bacteremia because of lack of treatment options and delayed diagnosis. $^{7,13,18,20}$

A previous study demonstrated that PBS was associated with 14-day mortality from CRE bacteremia or non-bacteremia CRE infections, and that a PBS over 2 was associated with mortality. ${ }^{21}$ Similarly, we observed that PBS was associated with one-week mortality in patients with CRGNB and VRE bacteremia. The mean PBS was $2.05 \pm 2.75$ in the survival group and $3.75 \pm$ 3.42 in the death group. Additionally, the SOFA score was also associated with early (one-week) mortality from CRGNB or VRE bacteremia.

Why a positive FUBC is a significant risk factor for early and in-hospital mortality is unclear. Some studies of gramnegative bacteremia revealed that FUBCs are not mandatory in patients that do not develop severe bacteremia. ${ }^{22,23}$ While the reason behind this is also unknown, it is possible that a larger bacterial burden may result in positive FUBCs, which may affect mortality.

The present findings indicate that several clinical variables, which include bacteremia of respiratory origin, malignancy, initial severity, initial hypotension, initial mechanical ventilation (MV), a high CRP level, low albumin level, longer time to active treatment, and non-negative conversion of FUBCs, could be predictors for mortality from CRGNB or VRE bacteremia.

This study has several strengths. First, we focused only on bacteremic cases and utilized data from a large number of patients $(\mathrm{N}=171)$. Although previous studies tried to identify risk factors of CRE or VRE bacteremia, many studies included carriers or rare studies included significant risk factors. This may be because there were few bacteremia cases. ${ }^{13,18,19}$ Our study overcame this limitation because of the relatively large number of patients and the identification of significant risk factors, including positive FUBC and PBS.
Second, this study analyzed severity indices together and then analyzed initial clinical variables and significant severity indices together. This approach was objective and reliable. Currently, the management of bacteremia is determined largely by clinical judgment. Some clinicians require initial variables and severity indices that can predict acute and 28-day mortality to improve treatment outcomes.

Third, this study used real-world data. Although MDR organisms are a considerable problem in clinics, most studies of MDR pathogens have involved laboratory data or select groups. ${ }^{20,24,25}$ Our study was not a randomized controlled study, but we included actual (and heterogeneous) patients. The data have real-world value.

This study also has several limitations. First, due to the retrospective nature of the study, there may have been bias during data collection. Given that this study constituted exploratory research without an exact calculation of sample size in a statistical manner, the study could be insufficiently powered to detect weaker, but potential clinically significant effects. Nevertheless, our study population comprised 171 cases and was relatively large considering that only resistant pathogens in true bacteremia cases were included. Therefore, our study still had some statistical power for detection.

Second, we did not analyze CPE pathogens separately or compare differences between organisms. A prior study investigated VRE carriers of E. faecalis. ${ }^{15}$ It is possible that CPE pathogens had different infection patterns that affected mortality. In contrast, other studies showed no difference between CPE and non-CPE infections. ${ }^{1,3}$

Third, we did not explore treatment aspects in detail. This was due to the lack of information of antibiotics prescribed, dose, and total therapy time. All these factors could influence mortality. However, our intent was to reveal the characteristics that can determine mortality at first glance; therefore, we did not focus on treatment aspects. All the patients in our study were treated with effective antibiotics for at least one day (24h), and we accounted for the time when the effective antibiotics started. There is debate concerning which antibiotics are the most effective in $\mathrm{CRGNB}^{26}$ or VRE. ${ }^{7}$ This uncertainty hindered the exploration of treatment.

\section{Conclusion}

In patients with CRGNB or VRE bacteremia, PBS was the most important initial severity score for predicting oneweek mortality. Univariate analysis revealed that 
hospitalization over 90 days, liver cirrhosis, respiratory origin or central line-related bacteremia, initial hypotension, mechanical ventilation, urea $>22 \mathrm{mg} / \mathrm{dL}, \mathrm{CRP}>15$ $\mathrm{m} / \mathrm{dL}, \mathrm{PBS}$, and positive FUBC were significant factors predicting one-week mortality. The multivariate analysis revealed that $\mathrm{PBS}$ and positive FUBC remained statistically significant.

Concerning 28-day mortality, bacteremia of respiratory origin, bacteremia of non-genitourinary origin, positive FUBC, initial hypotension, mechanical ventilation, initial WBC $>120 \times 103 \mathrm{~mm}^{3}$ or less than $<4 \times 103 \mathrm{~mm}^{3}$, platelet $<150 \times 10^{3} \mathrm{~mm}^{3}$, albumin $<3.5 \mathrm{~g} / \mathrm{dL}$, creatinine $>1.2 \mathrm{~mm} /$ $\mathrm{h}$, and BUN $>22 \mathrm{mg} / \mathrm{dL}$ were significant risk factors. In multivariate analysis, bacteremia of respiratory origin and positive FUBC were significantly related with 28-day mortality. Further multicenter, large-scale studies will be necessary to reduce and predict mortality from CRGNB or VRE bacteremia.

\section{Ethics Approval}

This study was approved by the Institutional Review Board of Gil Medical Center (GDIRB2020-017).

Institutional review board confirmed that this study did not need patients' consent because of lack of personal information in data. All of the data confidentiality was kept by the researcher according to the institutional protocols. This study followed the consolidated standards of reporting trials (CONSORT) guidelines which complies with the declaration of Helsinki.

\section{Disclosure}

All authors report no conflicts of interest in this work.

\section{References}

1. Britt NS, Ritchie DJ, Kollef MH, et al. Clinical epidemiology of carbapenem-resistant gram-negative sepsis among hospitalized patients: shifting burden of disease? Am J Infect Control. 2018;46 (10):1092-1096. doi:10.1016/j.ajic.2018.03.013

2. Hidron AI, Edwards JR, Patel J, et al. NHSN annual update: antimicrobial-resistant pathogens associated with healthcare-associated infections: annual summary of data reported to the National Healthcare Safety Network at the Centers for Disease Control and Prevention, 2006-2007. Infect Control Hosp Epidemiol. 2008;29 (11):996-1011. doi:10.1086/591861.

3. Livorsi DJ, Chorazy ML, Schweizer ML, et al. A systematic review of the epidemiology of carbapenem-resistant Enterobacteriaceae in the United States. Antimicrob Resist Infect Control. 2018;7(1):55. doi:10.1186/s13756-018-0346-9.

4. Centers of Korea disease control. Infectious disease portal Secondary Infectious disease portal 2020. http://www.cdc.go.kr/npt/biz/npp/ist/ simple/simplePdStatsMain.do. Accessed September 28, 2020.
5. Amit S, Mishali H, Kotlovsky T, Schwaber MJ, Carmeli Y. Bloodstream infections among carriers of carbapenem-resistant Klebsiella pneumoniae: etiology, incidence and predictors. Clin Microbiol Infect. 2015;21(1):30-34. doi:10.1016/j.cmi.2014.08.001.

6. Nordmann P, Cuzon G, Naas T. The real threat of Klebsiella pneumoniae carbapenemase-producing bacteria. Lancet Infect Dis. 2009;9 (4):228-236. doi:10.1016/S1473-3099(09)70054-4.

7. Prematunge $\mathrm{C}$, MacDougall $\mathrm{C}$, Johnstone J, et al. VRE and VSE bacteremia outcomes in the era of effective VRE therapy: A systematic review and meta-analysis. Infect Control Hosp Epidemiol. 2016;37(1):26-35. doi:10.1017/ice.2015.228.

8. McConville TH, Sullivan SB, Gomez-Simmonds A, Whittier S, Uhlemann A-C, Lazzeri C. Carbapenem-resistant Enterobacteriaceae colonization (CRE) and subsequent risk of infection and 90-day mortality in critically ill patients, an observational study. PLoS One. 2017;12(10):e0186195. doi:10.1371/journal. pone. 0186195

9. World Health Organization. Expert Committee on the International Classification of Diseases $M$, World Health Organization. Development of E, Health Statistical Services U. Expert Committee on the International Classification of Diseases, 10th Revision, Second Meeting, Geneva, November 23-27 1987 [Report]. Geneva, Switzerland: World Health Organization; 1987.

10. Institute CaLS. Performance Standards for Antimicrobial Susceptibility Testing. 30 ed. Vol. 296. 2020.

11. Poon LM, Jin J, Chee YL, et al. Risk factors for adverse outcomes and multidrug-resistant Gram-negative bacteraemia in haematology patients with febrile neutropenia in a Singaporean university hospital. Singapore Med J. 2012;53(11):720-725.

12. Oliveira PMN, Buonora SN, Souza CLP, et al. Surveillance of multidrug-resistant bacteria in pediatric and neonatal intensive care units in Rio de Janeiro State, Brazil. Rev Soc Bras Med Trop. 2019;52: e20190205. doi:10.1590/0037-8682-0205-2019.

13. Han SH, Chin BS, Lee HS, et al. Vancomycin-resistant enterococci bacteremia: risk factors for mortality and influence of antimicrobial therapy on clinical outcome. $J$ Infect. 2009;58(3):182-190. doi:10.1016/j.jinf.2009.01.013.

14. Hefazi M, Damlaj M, Alkhateeb HB, et al. Vancomycin-resistant Enterococcus colonization and bloodstream infection: prevalence, risk factors, and the impact on early outcomes after allogeneic hematopoietic cell transplantation in patients with acute myeloid leukemia. Transpl Infect Dis off J Transplant Soc. 2016;18(6):913-920. doi:10.1111/tid.12612.

15. Hayakawa K, Marchaim D, Martin ET, et al. Comparison of the Clinical Characteristics and Outcomes Associated with Vancomycin-Resistant Enterococcus faecalis and VancomycinResistant E. faecium Bacteremia. Antimicrob Agents Chemother. 2012;56(5):2452-2458. doi:10.1128/AAC.06299-11.

16. Zhang Y, Wang Q, Yin Y, et al. Epidemiology of carbapenem-resistant Enterobacteriaceae infections: report from the China CRE Network. Antimicrob Agents Chemother. 2018;62:2. doi:10.1128/ AAC.01882-17.

17. Ghafur A, Devarajan V, Raja T, et al. Monotherapy versus combination therapy against carbapenem-resistant Gram-negative bacteria: A retrospective observational study. Indian Journal of Cancer. 2016;53 (4):592-594. doi:10.4103/0019-509X.204767.

18. Satlin MJ, Chen L, Patel G, et al. Multicenter clinical and molecular epidemiological analysis of bacteremia due to carbapenem-resistant Enterobacteriaceae (CRE) in the CRE epicenter of the United States. Antimicrob Agents Chemother. 2017;61(4):4. doi:10.1128/ AAC.02349-16.

19. Li C, Li Y, Zhao Z, Liu Q, Li B. Treatment options and clinical outcomes for carbapenem-resistant Enterobacteriaceae bloodstream infection in a Chinese university hospital. J Infect Public Health. 2019;12(1):26-31. doi:10.1016/j.jiph.2018.08.002. 
20. Cai Y, Seah CL, Leck H, et al. Rapid antibiotic combination testing for carbapenem-resistant Gram-negative bacteria within six hours using ATP bioluminescence. Antimicrob Agents Chemother. 2018;62(9):e00183-18. doi:10.1128/AAC.00183-18.

21. Henderson H, Luterbach CL, Cober E, et al. The Pitt Bacteremia Score Predicts Mortality in Nonbacteremic Infections. Clin Infect Dis off Publ Infect Dis Soc Am. 2020;70(9):1826-1833. doi:10.1093/cid/ ciz528.

22. Shi H, Kang C-I, Cho SY, Huh K, Chung DR, Peck KR. Follow-up blood cultures add little value in the management of bacteremic urinary tract infections. Eur J Clin Microbiol Infect Dis. 2019;38 (4):695-702. doi:10.1007/s10096-019-03484-4.

23. Canzoneri CN, Akhavan BJ, Tosur Z, Andrade PEA, Aisenberg GM. Follow-up blood cultures in Gram-negative bacteremia: are they needed? Clin Infect Dis. 2017;65(11):1776-1779. doi:10.1093/cid/ cix 648 .
24. Pal A, Tripathi A. 4-Chloromercuribenzoic acid enhances carbapenem sensitivity among pathogenic Gram negative bacteria by altering bla, adeB and ompC expression. J Infect Public Health. 2020;13 (5):806-814. doi:10.1016/j.jiph.2019.11.008.

25. Rodríguez EA, Garzón LM, Gómez ID, Jiménez JN. Multidrug resistance and diversity of resistance profiles in carbapenem-resistant Gram-negative bacilli throughout a wastewater treatment plant in Colombia. J Glob Antimicrob Resist. 2020;22:358-366. doi:10.1016/j.jgar.2020.02.033.

26. Papst L, Beović B, Pulcini C, et al. Antibiotic treatment of infections caused by carbapenem-resistant Gram-negative bacilli: an international ESCMID cross-sectional survey among infectious diseases specialists practicing in large hospitals. Clin Microbiol Infect. 2018;24(10):1070-1076. doi:10.1016/j.cmi.2018.01.015.

\section{Publish your work in this journal}

Infection and Drug Resistance is an international, peer-reviewed openaccess journal that focuses on the optimal treatment of infection (bacterial, fungal and viral) and the development and institution of preventive strategies to minimize the development and spread of resistance. The journal is specifically concerned with the epidemiology of antibiotic resistance and the mechanisms of resistance development and diffusion in both hospitals and the community. The manuscript management system is completely online and includes a very quick and fair peerreview system, which is all easy to use. Visit http://www.dovepress.com/ testimonials.php to read real quotes from published authors. 